\title{
The Role of Magnetic Reconnection in Solar Activity
}

Spiro K. Antiochos and C. Richard DeVore

Naval Research Laboratory, Washington, D. C.

Short title: MAGNETIC RECONNECTION AND SOLAR ACTIVITY 


\begin{abstract}
We argue that magnetic reconnection plays the determining role in many of the various manifestations of solar activity. In particular, it is the trigger mechanism for the most energetic of solar events, coronal mass ejections and eruptive flares. We propose that in order to obtain explosive eruptions, magnetic reconnection in the corona must have an "on-off" nature, and show that reconnection in a sheared multi-polar field configuration does have this property. Numerical simulation results which support this model are presented, and implications for coronal mass ejections/eruptive flare prediction are discussed.
\end{abstract}




\section{INTRODUCTION}

Magnetic reconnection is widely believed to be the most important process by which magnetic fields transfer energy to plasmas and, consequently, has been invoked as the explanation for a multitude of phenomena observed in space, astrophysical, and laboratory plasmas. Reconnection is the most likely mechanism by which the Sun's magnetic field powers the solar atmosphere, the chromosphere and corona. Reconnection is also the process by which the solar wind flow couples to planetary magnetospheres, and is the key process behind the eventual transfer of this energy to the Earth's ionosphere and lower atmosphere. Hence, it would not be unjustified to describe the ISTP as a program to study the effects of reconnection on the Sun-Earth Connection.

Reconnection is particularly attractive as an explanation for solar variability and activity because of the potential for both rapid dissipation of magnetic energy and the accompanying strong plasma dynamics. In general, one can categorize the energy release due to reconnection as taking four distinct forms, all of which are believed to occur commonly in the solar atmosphere. First, reconnection can result in direct plasma heating. This has been proposed as the process that heats both the non-flare [e.g., Parker, 1972, 1979, 1983; Sturrock and Uchida, 1981; van Ballegooijen, 1986; Antiochos, 1990] and flare corona [e.g., Sweet, 1958; Parker, 1963; Petschek, 1964; Carmichael, 1964; Sturrock, 1966] Second, reconnection can produce strong mass motions, the so-called reconnection jets. These have been proposed [Karpen, Antiochos, and DeVore, 1995, 1996, 1998] as the explanation for a large variety of transient dynamic phenomena ranging from spicules [e.g., Blake and Sturrock, 1985] to explosive events [Dere et al., 1991] to large surges and sprays [e.g., Rust, 1968; Herant et al., 1991; Schmeider et al., 1994; Yokohama and Shibata, 1996] and X-ray jets [Shibata et al., 1992]. Third, reconnection can lead to the acceleration of non-thermal particles. This process has been proposed as the explanation for the electron beams that give rise to the hard X-ray emission in flares [e.g., Sturrock, 1980 and references therein]. Finally, reconnection can 
produce MHD waves. This process has been proposed as the explanation for coronal heating [e.g., Falconer et al., 1997] and for accelerating the solar wind [e.g., Parker, 1988; Mullan, 1990]. In fact, it is difficult to find a coronal phenomena that has not been attributed to magnetic reconnection!

Rather than considering one of these direct effects of reconnection, we discuss in this paper one of the most interesting and more subtle roles of reconnection - the initiation of major disruptions of the Sun's magnetic field. Large magnetic disruptions are generally observed as a coronal mass ejection (CME) and a flare associated prominence/filament eruption. We will refer to them as CME/eruptive flare (EF). These events are the most energetic (up to $10^{33}$ ergs) and destructive of solar disturbances. They are the main drivers of space weather and are a focus of study of the ISTP program. The May 21998 event, for example, was observed by SOHO/ISTP to consist of a filament eruption, fast $\mathrm{CME}(1,000 \mathrm{~km} / \mathrm{s})$ and an X-class flare that produced a strong proton storm at Earth and the strongest geomagnetic activity in recent years $\left(K_{p}=9\right)$. CME/EFs will be of particular interest in the upcoming maximum.

In addition to their key role in driving space weather, CME/EFs are important from the viewpoint of understanding basic space-plasma physics. They are the classic manifestation of solar activity, and the mechanism for their initiation and evolution has long been a central issue of solar physics research [e.g., Sturrock, 1980]. Space observations such as those from Yohkoh and SOHO have led to much progress in understanding magnetic disruptions, and a general framework for these events has emerged. A CME/EF is believed to consist of four steps: (1.) Stressed magnetic field slowly emerges from below the photosphere building up the free energy in the corona quasi-statically. The emerged field may be further stressed by the convective and rotational photospheric motions. (2.) Some triggering mechanism destroys the magnetic equilibrium of the corona, resulting in an explosive instability or loss of equilibrium. (3.) The magnetic field erupts outward, accelerating and ejecting plasma into the 
heliosphere. (4.) The field closes back down via reconnection to a less stressed state, heating the plasma and producing the intense X-ray burst.

In this four-step picture, a CME/EF can be thought of as simply the method by which the Sun rids itself of magnetic stress. Since the coronal conductivity is so high, the Sun has no choice but to eject any large-scale magnetic stress into the heliosphere. Note also that in this picture, a CME and an eruptive flare are part of a single phenomenon. The CME and prominence/filament eruption constitute step (3.), the opening of the field. It should be emphasized that by opening we don't mean that the field actually disconnects from the Sun, only that the field becomes dynamic and expands outward without limit. During this step the free magnetic energy is used primarily to accelerate plasma and lift it against gravity. The $\mathrm{X}$-ray or $\mathrm{H}_{\alpha}$ flare constitutes step (4.), the closing of the field. During this step the energy is used primarily to heat magnetically confined plasma and to accelerate nonthermal particles.

As with any astrophysical phenomenon, one can always find CMEs and flares that appear to be exceptions to the standard picture described above. Many CMEs, especially high-latitude slow ones, have no detectable X-ray emission. In this case the reconnection during step (4.) is presumably very slow, resulting in minimal heating. Much more difficult to reconcile, however, is the observation that intense X-ray flares sometimes occur with no apparent magnetic field opening i.e., no CME or prominence/filament eruption. Strong flare heating is widely believed to be due to fast reconnection, which generally involves the formation of a current sheet. It is the opening of the field during (3.) that forms a large current sheet, thereby, allowing rapid reconnection to occur during (4.). In order to account for non-eruptive flares, it must be that either the field does erupt but produces undetectable plasma motions — this seems unlikely — or else large current sheets can form and fast reconnection can occur even in fully closed bipolar configurations. There are good arguments, however, against the formation of current sheets in such a magnetic topology [e.g., van Ballejooigen, 
1985; Antiochos, 1987], furthermore reconnection is generally believed to be inhibited by line-tying effects for such topologies [Dahlburg, Antiochos, and Zang, 1991; Velli, Einaudi, and Hood, 1993]. More observational and theoretical work is clearly needed on the issue of apparently non-eruptive flares.

In this paper we adopt the standard 4-step picture above for a CME/EF and focus on Step (2.), the triggering mechanism. Determining the flare trigger has long been one of the outstanding problems in solar physics [e.g., Sturrock, 1980]. In addition, it is clearly necessary to understand the triggering process if we are ever to develop physics-based CME and eruptive flare prediction schemes. We argue below that magnetic reconnection in a multi-flux magnetic topology is this long-sought-after triggering mechanism.

\section{THEORETICAL RESULTS}

Over the years many models have been proposed for the initiation of CMEs and EFs, the large majority of which are magnetically driven [e.g., Sturrock, 1989; Moore and Roumeliotis, 1992; Mikic and Linker, 1994; Antiochos, DeVore, and Klimchuk, 1998], although models in which gas buoyancy effects are important [e.g., Low, 1994; Wolfson and Dlamini, 1997] have also been discussed. Since the solar corona is generally measured to have a low plasma beta $<10^{-2}$, it is almost certain that the energy for the eruption is stored in the magnetic field. This is especially true for the very energetic, fast eruptions which can have speeds exceeding $2000 \mathrm{~km} / \mathrm{s}$ - of order the Alfven speed, far larger than coronal sound speeds.

In recent years, however, a major obstacle to the magnetic models has been pointed out by Aly and Sturrock. Their argument proceeds as follows. Aly (1984) in a landmark paper proved that the energy of any force-free magnetic field configuration in the corona is bounded above. This result implies that if the coronal field is stressed by photospheric motions, the energy of the field will not continue rising indefinitely even if the motions 
continue indefinitely. Aly showed that, in fact, the energy has an upper bound that is only of order the energy of the potential magnetic field with the same normal flux distribution at the photosphere. For example, a dipole field is bounded above by twice the potential field energy [Aly, 1984]. The physical reason for the energy bound is straightforward. The corona has an infinite volume, and so if even the footpoint motions become infinitely large, the field can simply expand outward toward infinity, thereby, keeping its energy finite. Note that this argument is equally valid if the magnetic stress is due to emergence from below rather than footpoint motions. As long as the evolution is quasi-static, the magnetic free energy and also the electric current, or magnetic stress, in the corona must have a strict upper bound [e.g., Aly, 1984; Finn and Chen, 1990]. Therefore, one must be careful in specifying boundary conditions for the corona at its photospheric base. For example, one is not free to specify arbitrarily the current entering the corona from the photospheric. In general, the safest and most physical boundary conditions are to specify footpoint motions, which is our approach in the simulations described below.

Building upon this work, Aly (1991) presented arguments that if the coronal magnetic field undergoes a quasi-static evolution, which is a good approximation for photospheric driving motions, then the least upper bound on its energy is the open field configuration in which every field line is stretched out to infinity. Sturrock (1991) independently presented similar arguments. Although their arguments do not constitute a rigorous proof, they are quite convincing and appear to be in good agreement with every numerical simulation performed to date [e.g., Roumeliotis, Antiochos, and Sturrock, 1994; Mikic and Linker, 1994; Amari et al., 1996], (but see Wolfson and Low (1992) for a possible counter example). The Aly-Sturrock energy limit also seems physically intuitive. The basic assumption is that the coronal field is always free to lower its energy by an ideal outward expansion, if such an expansion is energetically favorable. Consequently, it should not be possible to build up quasi-statically significant energy 
above the fully open state, because as soon as the magnetic energy tries to exceed the open value, the field would simply expand to the open state. Once the field is open, further footpoint motions have no effect other than to propagate Alfven waves out into the heliosphere.

Although the energy limit seems perfectly reasonable, it also seems completely incompatible with $\mathrm{CME} / \mathrm{EF}$ observations. If these events are magnetically driven, the energy in the pre-eruption magnetic field must be considerably larger than the open field state formed during the eruption, contrary to the energy limit. It is often claimed that one can circumvent the energy limit by simply opening part of the field. For example, one can leave the very low-lying field closed and open only above a certain height. We believe that this argument is spurious. While it is true that in their papers Aly and Sturrock concentrated on the fully open field case, as we discussed above, their result is based on quite general arguments that should hold even for a partial opening of the field. The basic point is that a slowly driven magnetic field is unlikely to erupt explosively to an open state that it can also reach by a process of ideal expansion. Indeed, EIT and LASCO/C1 observations from SOHO show that slow expansion is a general feature of the coronal magnetic field. Furthermore, we believe that most CMEs, which are typically slow and accelerate only up to slow-solar wind speeds, also represent merely a slow expansion of the field. On the other hand, there is no doubt that explosive eruptions do sometimes occur.

\section{NUMERICAL RESULTS}

An attractive solution to this apparent contradiction between theory and observation is to invoke reconnection. It seems likely that the extra freedom in the possible evolution of the field afforded by reconnection can somehow circumvent the energy limit. This is exactly what happens in 2.5D geometries. Mikic and Linker (1994) showed that footpoint shearing in a system with azimuthal symmetry and finite 
resistivity leads to the formation of disconnected plasmoids that are expelled rapidly from the corona with a significant energy drop. We emphasize again that, contrary to common misconception, the eruption in this case is not due the fact that the field opens only partially. The eruption occurs because the field opens to a state that it cannot access by an ideal expansion. The plasmoid carries off a large fraction of the shear of the closed field lines, so that the final state of the system is one in which the closed region contains much less shear than was originally imparted to this region by the footpoint motions. The field could not erupt to the state in which the shear in the closed field region stayed fixed.

This discussion leads to the main conclusion of this paper: the whole eruption process is controlled by the nature of magnetic reconnection in the corona. In order for plasmoid formation to result in an explosive eruption, the formation and hence the reconnection must proceed rapidly. The plasmoid must grow faster than the field expands outward. On the other hand, reconnection must not occur too easily, otherwise very little free energy could be built up. Therefore, to explain eruptions, magnetic reconnection in the solar corona must have a very particular form. It must have an "on-off" nature wherein it is basically off during step (1.) while the free energy is building up, but at a certain point switches on to a high value and stays on. It is this reconnection switch-on that is the flare trigger, step (2.).

Since plasmoid formation is only a $2.5 \mathrm{D}$ phenomenon, it is not clear, however, whether reconnection can also lead to an eruption in a full 3D system. The 3D analogue to the 2.5D configuration is the so-called tether-cutting model [Sturrock, 1989; Moore and Roumeliotis, 1991]. The basic geometry of this model can be seen in Plate 1, which shows the results of a 3D MHD simulation of photospheric stressing of a coronal field. This simulation is a fully time-dependent calculation of our model for prominence formation [Antiochos, Dahlburg, and Klimchuk, 1994; Antiochos, 1995], and corresponds to a $3 \mathrm{D}$ generalization of the $2.5 \mathrm{D} \mathrm{CME}$ calculations. The magnetic field in the figure is 
the result of applying a shear flow at the photosphere to an initially current-free dipole field. The plane of the photosphere in this simulation was taken to be a rectangle, part of which can be seen in the figure. The straight black line running lengthwise through the middle of the photospheric plane is the magnetic polarity reversal line. In agreement with observations [Schmeider et al., 1996], the shear was chosen to be spatially localized near the polarity reversal line. The form of this shear can be seen in the contours of the normal component of $B$ on the photospheric plane; for ease of viewing only one contour is shown on the far side of the bottom plane. Note that in the corona above this plane there are basically two types of field lines. Those originating in the shear zone (three thick lines) are stretched out and have concave up portions along their length, while those originating in the non-shear zone (three thin arrowed lines) appear to be dipolar. The mass of red lines in the center of the figure indicates where the magnetic field lines are concave up and represents the $\mathrm{H}_{\alpha}$ mass of the prominence.

The idea behind tether-cutting is that reconnection leads to eruption by redistributing the shear so that it becomes concentrated on the furthermost field lines at the edge of the sheared region. The field lines around the center of the figure decrease their shear, while those near the edges increase. Such a transfer of shear to the outermost field lines is the physical analogue to plasmoid formation in $2.5 \mathrm{D}$. If this shear transfer is sufficiently rapid, then eruption should occur. Hence, the 3D case is really not significantly different than the $2.5 \mathrm{D}$ system. In both cases the question of whether an eruption occurs comes down to the issue of the temporal behavior of reconnection in the solar corona

So far, our 3D simulations of tether-cutting show no evidence for an explosive eruption, which agrees with our previous work on the twisting of dipolar arcades [Dahlburg, Antiochos, and Zang, 1991]. However, this work is still in progress, and it may be that using different parameters, such as system size, form of shear, etc. will lead to eruption. Further studies of the tether-cutting model are needed before definitive 
conclusions can be reached.

Based on our work so far, however, we conjecture that the tether-cutting model does not lead to explosive eruptions. The basic reason for this result is that a bipolar field system as in Plate 1 is too simple topologically for fast reconnection to occur. We propose instead the "breakout" model [Antiochos, 1998; it Antiochos, DeVore, and Klimchuk, 1999] shown in Plate 2, which consists of a multi-polar field. The initial current-free field for this system is given by the vector potential:

$$
\vec{A}=\frac{\sin \theta}{r^{2}}+\frac{(3+5 \cos 2 \theta) \sin \theta}{2 r^{4}} \hat{\phi}
$$

This system has a more complex topology than that of Plate 1, it consists of four flux systems with separatrix surfaces and a null point in the corona, as shown in the first panel of Plate 2. There are now three neutral lines in the system, at solar latitudes of $\pm 45^{\odot}$ and at the equator. Note, however, that this field configuration arises from only four polarity regions on the photospheric surface, hence it is actually far simpler than what must be present in the real corona. Also it appears similar to what is often seen in the LASCO C1 images.

The effect of stressing this system by footpoint motions can be seen in Plate 2, which present the results from one of our 2.5D MHD simulations. For this case we selected the inner flux system (straddling the equator), as the one to shear. Again the shear is concentrated near the neutral line. The thick dark lines in Plate 2 correspond to the sheared prominence field lines of Plate 1. Basically, we have embedded the sheared arcade of Plate 1 into a more global field topology. As a result of being sheared, the inner flux system overlying the equator expands outward, just as the single arcade in Plate 1 expands outward. But now it begins to interact with the neighboring systems. As a result of this expansion the null point in the corona deforms into a neutral sheet, similar to the process originally proposed by Syrovatskii (1981). Eventually, this neutral sheet becomes sufficiently thin that reconnection begins. We see in the last panel 
of Plate 2 that some of the field lines that used to be in the inner and outer flux systems have transferred over to the sides. Once this reconnection starts it continues to accelerate because there are fewer unsheared field lines holding down the sheared ones. The rapidly accelerating transfer of flux from above the sheared field allows this field to "break out" and erupt open.

Note that our breakout model yields a very natural process for obtaining an "on-off" type of reconnection in the corona. In fact, we find that once the reconnection switches on, the rate becomes too large for our ideal code to simulate, but the essential result has been shown - in a multi-flux system reconnection will have the correct time-dependence to explain the initiation of CME/EFs, step (2.). Hence, we conclude that reconnection is responsible not only for releasing a large fraction of the flare energy during step (4.), it is also responsible for getting the whole event going.

Now that we have a likely candidate for the trigger mechanismm, it is interesting to consider its use as a possible CME/EF predictor. There are two distinguishing features of the breakout model. One is that a multi-polar configuration is needed for eruption. In fact, it is well known that complexity is needed to obtain eruptive flares, and magnetic complexity is already one of the main criteria being used for prediction. The second feature is that reconnection should occur high up in the corona before the eruption. We believe that it would be highly informative to use the resources of the ISTP to search for this reconnection during the upcoming solar maximum.

Acknowledgments. This work was supported in part by NASA and ONR. 


\section{References}

Aly, J. J., On some properties of force-free magnetic fields in infinite regions of space, Astrophys. J., 283, 349, 1984.

Aly, J. J., How much energy can be stored in a three-dimensional force-free magnetic field?, Astrophys. J., 374, L61, 1991.

Amari, T., Luciani, J. F., Aly, J. J., and Tagger, M., Very Fast Opening of a Three-dimensional Twisted Magnetic Flux Tube, Astrophys. J., 466, L39, 1996.

Antiochos, S. K., The topology of force-free magnetic fields and its implications for coronal activity, Astrophys. J., 312, 886, 1987.

Antiochos, S.K., Heating of the corona by magnetic singularities, J. Italian Astron. Soc., 61, $369,1990$.

Antiochos, S. K., Dahlburg, R. B., and Klimchuk, J. A., The magnetic field of solar prominences, Astrophys. J., 420, L41, 1994.

Antiochos, S. K., Solar drivers of space weather, Astron. Soc. Pac. Conf. Series, 95, 1, 1995.

Antiochos, S.K., The magnetic topology of solar eruptions, Astrophys. J., 502, L181, 1998.

Antiochos, S. K., DeVore, C. R., and Klimchuk, J. A., A model for solar coronal mass ejections, Astrophys. J., 510, in press, 1999.

Blake, M. L., and Sturrock, P. A., Spicules and surges, Astrophys. J., 290, 359, 1985.

Carmichael, H., A process for flares, in AAS-NASA Symp. on Physics of Solar Flares, edited by W. Ness, p. 451, NASA SP-50, Washington, DC, 1964.

Dahlburg, R. B., Antiochos, S. K., and Zang, T. A., Dynamics of solar coronal magnetic fields, Astrophys. J., 383, 420, 1991.

Dere, K. P., Bartoe, J.-D. F., Brueckner, G. E., Ewing, J., and Lund, P., Explosive events and magnetic reconnection in the solar atmosphere, J. Geophys. Res., 96, 9399, 1991.

Falconer, D. A. et al., Neutral-line magnetic shear and enhanced coronal heating in solar active regions, Astrophys. J., 482, 519, 1997.

Finn, J. M., and Chen, J., Equilibrium of solar coronal arcades, Astrophys. J., 349, 345, 1990.

Herant, M., Pardo, F., Spiller, E, and Golub, L., Flares observed by the normal incidence 
X-ray telescope on 1989 September 11, Astrophys. J., 376, 797, 1991.

Karpen, J. T., Antiochos, S. K., and DeVore, C. R., The role of magnetic reconnection in chromospheric eruptions, Astrophys. J., 450, 422, 1995.

Karpen, J. T., Antiochos, S. K., and DeVore, C. R., Reconnection driven current filamentation in solar arcades, Astrophys. J., 460, L73, 1996.

Karpen, J. T., Antiochos, S. K., and DeVore, C. R., Dynamic responses to magnetic reconnection of solar arcades, Astrophys. J., 495, 491, 1998.

Low, B.C., Magnetohydrodynamic processes in the solar corona: flares, coronal mass ejetcions, and magnetic helicity, Phys. Plasmas, 1, 1684, 1994.

Mikić, Z. and Linker, J. A., Disruption of coronal magnetic field arcades, Astrophys. J., 430, 898, 1994.

Moore, R. L. and Roumeliotis, G., Triggering of eruptive flares: destabilization of the preflare magnetic field configuration, in Eruptive Solar Flares, edited by Z. Svestka, B. V. Jackson, and M. E. Machado, p. 69, Springer, Berlin, 1992.

Mullan, D. J., Sources of the solar wind - What are the smallest-scale structures? Astron. Astrophys., 248, 256, 1990.

Parker, E. N., The solar flare phenomenon and the theory of reconnection and annihilation of magnetic fields, Astrophys. J. Supp., 8, 177, 1963.

Parker, E. N., Cosmical Magnetic Fields, Clarendon Press, Oxford, U.K., 1979.

Parker, E. N., Magnetic neutral sheets in evolving fields. I - General theory. II - Formation of the solar corona, Astrophys. J., 264, 642, 1983.

Parker, E. N., Nanoflares and the solar X-ray corona, Astrophys. J., 330, 474, 1988.

Petschek, H. E., Magnetic field annihilation, in AAS-NASA Symp. on Physics of Solar Flares, edited by W. Ness, p. 425, NASA SP-50, Washington, DC, 1964.

Rust, D. M., Chromospheric explosions and satellite sunspots, in Structure and Development of Solar Active Regions (IAU Symposium 35), edited by K. O. Kiepenheuer, p. 77, Reidel, Dordrecht, 1968.

Schmieder, B., Golub, L., and Antiochos, S. K., Comparison between cool and hot plasma behaviors of surges, Astrophys. J., 425, 326, 1994. 
Schmieder, B., Demoulin, P., Aulanier, G., and Golub, L. Differential Magnetic Field Shear in an Active Region, Astrophys. J., 467,, 881, 1996.

Shibata, K., et al., Observations of X-ray jets with the Yohkoh Soft X-ray Telescope, Publ. Astron. Soc. Japan, 44, L173, 1992.

Sturrock, P. A., A Model of the High Energy Phase of Solar Flares, Nature, 211, 695, 1966.

Sturrock, P.A., Solar Flares, Colorado Assoc. Univ. Press, Boulder, CO., 1980.

Sturrock, P. A. and Uchida, Y., Coronal heating by stochastic magnetic pumping, Astrophys. J., $246,331,1981$.

Sturrock, P. A., The role of eruption in solar flares, Solar Phys., 121, 387, 1989.

Sturrock, P. A., Maximum energy of semi-infinite magnetic field configurations, Astrophys. J., 380, 655, 1991.

Sweet, P. A., in Electromagnetic Phenomena in Cosmic Physics, edited by B. Lehnert, pp. 123-34, Cambridge Univ. Press, New York, 1958.

Syrovatskii, S. I., Pinch sheets and reconnection in astrophysics, Ann. Rev. Astron. Aatrophys., 19, 163, 1981.

van Ballegooijen, A. A., Electric currents in the solar corona and the existence of magnetostatic equilibrium, Astrophys. J., 298, 421, 1985.

van Ballegooijen, A. A., Cascade of magnetic energy as a mechanism of coronal heating, Astrophys. J., 311, 1001, 1986.

Velli, M., Einaudi, G., and Hood, A. W., Ideal kink instabilities in line-tied coronal loops Growth rates and geometrical properties, Astrophys. J., 350, 428, 1990.

Wolfson, R. and Low, B. C., Energy buildup in sheared force-free magnetic fields Astrophys. J., 391, 353, 1992.

Wolfson, R and Dlamini, B, Cross-field currents: An energy source for coronal mass ejections? Astrophys. J., 483, 961, 1997.

Yokoyama, T., and Shibata, K., Numerical simulation of solar coronal X-Ray jets based on the magnetic reconnection model, Publ. Astron. Soc. Japan, 48, 353, 1996.

S. K. Antiochos, Code 7675, Naval Research Lab, Washington, DC 20375-5352. 
(e-mail: antiochos@nrl.navy.mil

C. R. DeVore, Code 6440, Naval Research Lab, Washington, DC 20375. (e-mail: devore@lcp.nrl.navy.mil)

Received 
Plate 1. Results from a 3D MHD simulation of our model for prominence/filament formation and of the tether cutting model for coronal mass ejections/ eruptive flares. The configuration shown is that of an initial current-free dipole field that has been sheared by photospheric motions. The outline of the bottom boundary of our simulation box is shown along with the photospheric neutral line. Contours of normal-magnetic-field magnitude are plotted on the bottom boundary plane. Three field lines (thick dark-blue) with footpoints in the shear region and three field lines (arrowed, light-blue) with footpoints outside this region are shown. Also plotted are contours of positive-upward curvature of magnetic field (mass of red lines), which corresponds to the possible location of the prominence mass.

Plate 2. Results from a 2.5D simulation of the breakout model for coronal mass ejections and eruptive flares. The first panel (upper left) shows the initial current-free configuration which consists of a sum of a dipolar and an octopolar field. Photospheric shearing is applied only near the equatorial neutral line. The next three panels show the field after 17.5, 19.7, and 21.7 hours into the evolution. All field lines are traced from exactly the same footpoint positions in all panels. Note that in the last panel the overlying unsheared field is reconnecting and moving to the side, thereby allowing the sheared flux to erupt outward. 
This figure "plate1.gif" is available in "gif" format from: http://arxiv.org/ps/astro-ph/9809161v1 
This figure "plate2.gif" is available in "gif" format from: http://arxiv.org/ps/astro-ph/9809161v1 